PROCEEDINGS OF THE

AMERICAN MATHEMATICAL SOCIETY

Volume 129, Number 1, Pages 37-43

S 0002-9939(00)05492-7

Article electronically published on July 27, 2000

\title{
APPROXIMATING DISCRETE VALUATION RINGS BY REGULAR LOCAL RINGS
}

\author{
WILLIAM HEINZER, CHRISTEL ROTTHAUS, AND SYLVIA WIEGAND
}

(Communicated by Wolmer V. Vasconcelos)

\begin{abstract}
Let $k$ be a field of characteristic zero and let $(V, \mathbf{n})$ be a discrete rank-one valuation domain containing $k$ with $V / \mathbf{n}=k$. Assume that the fraction field $L$ of $V$ has finite transcendence degree $s$ over $k$. For every positive integer $d \leq s$, we prove that $V$ can be realized as a directed union of regular local $k$-subalgebras of $V$ of dimension $d$.
\end{abstract}

\section{INTRODUCTION}

Suppose $(R, \mathbf{m})$ is a local Noetherian domain with fraction field $F$ and $(V, \mathbf{n})$ is a valuation domain that birationally dominates $R$ in the sense that $R$ is a subring of $V, V$ has fraction field $F$ and $\mathbf{n} \cap R=\mathbf{m}$. Classically, in the case where $R$ is essentially finitely generated over a field, local uniformization of $R$ along $V$, in algebraic terms, means the existence of a regular local domain $S$ between $R$ and $F$, such that $S$ is dominated by $V$ and is essentially finitely generated over $R$. If $R$ is a regular local ring and $P$ is a prime ideal of $R$, embedded local uniformization translates to the existence of $S$ as above having a prime ideal $Q$ with $Q \cap R=P$ and $S / Q$ a regular local domain.

The consideration of embedded local uniformization yields a representation of the valuation domain $V$ as a directed union of regular local rings. If $V / \mathbf{n}$ is algebraic over $R / \mathbf{m}$, then classical methods give a representation of $V$ as a directed union of regular local rings all having dimension equal to the dimension of $R$ [A, Lemma 12]. In this article we prove that certain rank-one discrete valuation rings (DVRs) can be represented as a directed union of regular local domains of dimension $d$ for every positive integer $d$ less than or equal to the dimension of $R$. We use a technique inspired by Nagata $\mathrm{N} 1$ and developed in our earlier work for the construction of new Noetherian domains [HRW1, HRW2, HRW3.

The classical approach for obtaining embedded local uniformization, introduced by Zariski in the 1940's [Z1], uses local quadratic transforms of $R$ along $V$. The

Received by the editors July 23, 1998 and, in revised form, March 22, 1999.

1991 Mathematics Subject Classification. Primary 13F30, 13H05; Secondary 13E05, 13G05, 13J05, 13J15.

Key words and phrases. Discrete rank-one valuation domain, étale extension, excellent ring, Henselization, local uniformization, regular local domain.

The authors thank the National Science Foundation and the National Security Agency for support for this research. In addition they are grateful for the hospitality and cooperation of Michigan State University, the University of Nebraska and Purdue University, where several work sessions on this research were conducted. 
first local quadratic transform of $R$ along $V$ is defined to be $R_{1}=R[\mathbf{m} / a]_{\mathbf{m}_{1}}$, where $a \in \mathbf{m}$ is such that $\mathbf{m} V=a V$ and $\mathbf{m}_{1}:=\mathbf{n} \cap R[\mathbf{m} / a] . R_{1}$ is also called the dilatation of $R$ by the ideal $\mathbf{m}$ along $V$ [N2, page 141]. More generally, if $I \subseteq \mathbf{m}$ is a nonzero ideal of $R$, the dilatation of $R$ by $I$ along $V$ is the $\operatorname{ring} R[I / a]_{\mathbf{m}_{1}}$, where $a \in I$ is such that $I V=a V$ and $\mathbf{m}_{1}=\mathbf{n} \cap R[I / a]$. It is straightforward to check that $R_{1}$ is uniquely determined by $R, V$ and the ideal $I$ [N2, page 141]. For each positive integer $i$, we define the $(i+1)$ th quadratic transform $R_{i+1}$ of $R$ along $V$ inductively: $R_{i+1}$ is the first local quadratic transform of $R_{i}$ along $V$. Then $R_{i+j}=\left(R_{i}\right)_{j}$ for all $i, j \geq 0$.

Using monoidal transforms (a special type of dilatation), S. Cutkosky in C1] and [C2 has recently done interesting work on birational factorization and local uniformization.

\subsection{Background remarks.}

(1) If $R$ is a regular local ring, it is well known that a quadratic transform $R_{1}$ of $R$ is again a regular local ring [N2, (38.1)].

(2) If $R$ is a pseudogeometric regular local domain of dimension 2 and $V$ is a valuation domain that birationally dominates $R$, a classical result of Zariski and Abhyankar asserts that $V=\bigcup_{n=1}^{\infty} R_{n}$ A Lemma 12].

(3) Suppose $R$ is a regular local ring of dimension $d \geq 3$. For certain valuation rings $V$ that birationally dominate $R$, the union $\bigcup_{n=1}^{\infty} R_{n}$ of the local quadratic transforms of $R$ along $V$ is strictly smaller than $V[\underline{S}$ (4.13)]. In many cases Shannon in $[\mathrm{S}$, (4.5), page 308] proves that $V$ is a directed union of iterated monoidal transforms of $R$, where a monoidal transform of $R$ is a dilatation of $R$ by a prime ideal $P$ for which $R / P$ is again regular.

(4) If $(V, \mathbf{n})$ is a DVR that birationally dominates a regular local ring $R$, then $\left.V=\bigcup_{n=1}^{\infty} R_{n}, 1\right]$ where $R_{n}$ is the $n$th quadratic transform of $R$ along $V$, page 336], [Z2, page 27-28].

A proof of item (4) goes as follows: a nonzero element $\eta$ of $V$ has the form $\eta=b / c$, where $b, c \in R$. If $(b, c) V=V$, then $b / c \in V$ implies $c V=V$. Since $V$ dominates $R$, it follows that $c R=R$, so $b / c \in R$ in this case. If $\eta=b / c$, with $b, c \in R$ and $(b, c) V=\mathbf{n}^{k}$, we prove by induction that $\eta \in R_{k}$. We have already done the case where $k=0$. Assume for every regular local domain $(S, \mathbf{p})$ birationally dominated by $V$, and every nonzero element $\beta / \gamma \in V$ with $\beta, \gamma \in S$, $(\beta, \gamma) V=\mathbf{n}^{j}$ and $0 \leq j<k$ we have $\beta / \gamma \in S_{j}$, where $S_{j}$ is the $j$ th iterated local quadratic transform of $S$ along $V$. Suppose $\beta, \gamma \in S, \beta / \gamma \in V$ with $(\beta, \gamma) V=\mathbf{n}^{k}$. Let $S_{1}=S[\mathbf{p} / a]_{\mathbf{p}_{1}}$, where $a \in \mathbf{p}$ is such that $\mathbf{p} V=a V$ and $\mathbf{p}_{1}:=\mathbf{n} \cap S[\mathbf{p} / a]$; that is, $S_{1}$ is the first local quadratic transform of $S$ along $V$. Then $\beta_{1}:=\beta / a$ and $\gamma_{1}:=\gamma / a$ are in $S_{1}$. Thus $a \in \mathbf{n}$ implies $\left(\beta_{1}, \gamma_{1}\right) V=\mathbf{n}^{j}$ where $0 \leq j<k$, so by induction

$$
\beta / \gamma=\beta_{1} / \gamma_{1} \in\left(S_{1}\right)_{j}=S_{j+1} \subseteq S_{k},
$$

which completes the proof.

In this article we prove the following theorem:

1.2 Main theorem. Let $k$ be a field of characteristic zero and let $(V, \mathbf{n})$ be a $D V R$ containing $k$ with $V / \mathbf{n}=k$. Assume that the fraction field $L$ of $V$ has finite transcendence degree $s$ over $k$. Then for every integer $d$ with $1 \leq d \leq s$, there

\footnotetext{
${ }^{1}$ It may happen that $R_{n}=V$ for some $n$, in which case $R_{n+i}=R_{n}$ for all $i \geq 0$.
} 
exists a nested family $\left\{C_{n}^{(\alpha)}: n \in \mathbb{N}, \alpha \in \Gamma\right\}$ of d-dimensional regular local $k$ subalgebras of $V$ such that $V$ is the directed union of the $C_{n}^{(\alpha)}$ and $V$ dominates each $C_{n}^{(\alpha)}$.

If the field $L$ is finitely generated over $k$, then $V$ is a countable union $\bigcup_{n=1}^{\infty} C_{n}$, where, for each $n \in \mathbb{N}$,

(1) $C_{n}$ is a d-dimensional regular local $k$-subalgebra of $V$,

(2) $C_{n}$ has fraction field $L$,

(3) $C_{n+1}$ dominates $C_{n}$ and

(4) $V$ dominates $C_{n}$.

We have the following corollary to the main theorem.

1.3 Corollary. Let $k$ be a field of characteristic zero and let $(R, \mathbf{m})$ be a local domain essentially of finite type over $k$ with coefficient field $k=R / \mathbf{m}$ and field of fractions L. Let $(V, \mathbf{n})$ be a DVR birationally dominating $R$ with $V / \mathbf{n}=k$. For every integer $d$ with $1 \leq d \leq s, s=\operatorname{trdeg}_{k}(L)$, there exists a sequence of $d$ dimensional regular local $k$-subalgebras $C_{n}$ of $V$ such that $V=\bigcup_{n=1}^{\infty} C_{n}$, and for each $n \in \mathbb{N}, C_{n+1}$ dominates $C_{n}$ and $V$ dominates $C_{n}$. Moreover $C_{n}$ dominates $R$ for all sufficiently large $n$.

Proof. By Theorem 1.2, $V=\bigcup_{n=1}^{\infty} C_{n}$, where the $C_{n}$ are regular local rings satisfying all the conditions of Corollary 1.3 except possibly that of dominating $R$. Since $R$ is essentially of finite type over $k$ and is dominated by $V$, it follows that $R$ is dominated by $C_{n}$ for all sufficiently large $n$.

1.4 Discussion. (1) If $L / k$ is finitely generated of transcendence degree $s$, then the fact that $V$ is a directed union of $s$-dimensional regular local rings follows from classical theorems of Zariski. The local uniformization theorem of Zariski [Z1] implies the existence of a regular local domain $(R, \mathbf{m})$ containing the field $k$ such that $V$ birationally dominates $R$. Since $k$ is a coefficient field for $V$, we have

(a) $k \hookrightarrow V \rightarrow V / \mathbf{n} \cong k$; thus $k$ is relatively algebraically closed in $L$.

(b) $R / \mathbf{m}=k$ (because $V$ dominates $R$ ).

(c) Every iterated local quadratic transform of $R$ along $V$ has dimension $s$.

Now by $1.1(4), V$ is a directed union of $s$-dimensional RLRs.

(2) If $d=1$, the main theorem is trivially true by taking each $C_{n}=V$. Thus if $L / k$ is finitely generated of transcendence degree $s=2$, then the theorem is saying nothing new.

(3) If $s>2$, then the classical local uniformization theorem says nothing about expressing $V$ as a directed union of $d$-dimensional RLRs, where $2 \leq d \leq s-1$. If $(S, \mathbf{p})$ is a local Noetherian domain containing $k$ and birationally dominated by $V$ with $\operatorname{dim}(S)=d<s$, then $S$ does not satisfy the dimension formula. It follows that $S$ is not essentially finitely generated over $k$ [M1 page 119].

We use the following notation in the proof of the main theorem.

1.5 Endpieces and related localized polynomial rings. Let $(R, \mathbf{m})$ be a local Noetherian domain with fraction field $F$. Let $y \in \mathbf{m}$ be a nonzero element, let $R^{*}=(\widehat{R,(y)})$ be the $(y)$-adic completion of $R$. Suppose $\tau_{1}, \ldots, \tau_{s} \in y R^{*}$ are regular element: 2 of $R^{*}$ that are algebraically independent over $K$. We consider

\footnotetext{
${ }^{2}$ We say an element of a ring is a regular element if it is not a zero divisor.
} 
the localized polynomial ring

$$
B_{0}:=R\left[\tau_{1}, \ldots, \tau_{s}\right]_{\left(\mathbf{m}, \tau_{1}, \ldots, \tau_{s}\right)} .
$$

It is clear that $B_{0}$ is a local Noetherian domain with $\operatorname{dim}\left(B_{0}\right)=\operatorname{dim}(R)+s$ such that $R^{*}$ dominates $B_{0}$ and $B_{0}$ dominates $R$.

For every $\gamma \in R^{*}$ and every $n>0$, we define the $n$ th-endpiece $\gamma_{n}$ with respect to $y$ of $\gamma$ to be

$$
\gamma_{n}:=\sum_{j=n+1}^{\infty} c_{j} y^{j-n}, \text { where } \gamma:=\sum_{j=1}^{\infty} c_{j} y^{j} \text { and each } c_{j} \in R
$$

In particular, we represent each of the $\tau_{i}$ by a power series expansion in $y$; we use these representations to obtain for each positive integer $n$ the $n$ th-endpieces $\tau_{i n}$ and corresponding $n$ th-localized polynomial ring $B_{n}$. For $1 \leq i \leq s$, and $\tau_{i}:=$ $\sum_{j=1}^{\infty} a_{i j} y^{j}$, where the $a_{i j} \in R$, we have

$$
\tau_{i n}:=\sum_{j=n+1}^{\infty} a_{i j} y^{j-n}, \quad B_{n}:=R\left[\tau_{1 n}, \ldots, \tau_{s n}\right]_{\left(\mathbf{m}, \tau_{1 n}, \ldots, \tau_{s n}\right)}, \text { for each } n \in \mathbb{N} .
$$

The definition of $B_{n}$ is independent of the representation of the $\tau_{i}$ as power series in $y$ HRW1, (2.3)]. The localized polynomial ring $B_{n+1}$ birationally dominates $B_{n}$. We define

$$
B:=\bigcup_{n=0}^{\infty} B_{n}=\varliminf_{\lim } B_{n} \quad \text { and } \quad A:=F\left(\tau_{1}, \ldots, \tau_{s}\right) \cap R^{*} .
$$

It is readily seen that $A$ birationally dominates $B$.

In the proof of our main theorem, we make use of the following result from previous work that we summarize as Theorem 1.6.

1.6 Theorem ([HRW1] or HRW2]). Suppose $(R, \mathbf{m})$ is a local Noetherian domain with fraction field $F, y \in \mathbf{m}$ is a nonzero element, and $\tau_{1}, \ldots, \tau_{s}$ are elements of the $(y)$-adic completion $R^{*}$ of $R$ that are algebraically independent over $R$. Then the following conditions are equivalent:

(1) The ring $R^{*}[1 / y]$ is flat over $B_{0}=R\left[\tau_{1}, \ldots, \tau_{s}\right]_{\left(\mathbf{m}, \tau_{1}, \ldots, \tau_{s}\right)}$.

(2) The ring $R^{*}[1 / y]$ is flat over $R\left[\tau_{1}, \ldots, \tau_{s}\right]$.

(3) The directed union $B:=\bigcup_{n=0}^{\infty} B_{n}$ is Noetherian.

Moreover, if these equivalent conditions hold, then the domain $A:=F\left(\tau_{1}, \ldots, \tau_{s}\right) \cap$ $R^{*}$ is equal to $B$, and thus both are Noetherian.

\section{Proof of the MAIN THEOREM}

Let $y \in \mathbf{n}$ be such that $y V=\mathbf{n}$. Then the $\mathbf{n}$-adic completion $\widehat{V}$ of $V$ is $k[[y]]$. Hence we have

$$
k[y]_{(y)} \subseteq V \subseteq k[[y]] .
$$

Then $V=L \cap k[[y]]$, for example, since $V \hookrightarrow k[[y]]$ is flat. Since $\operatorname{trdeg}_{k(y)} L=s-1$, there are $s-1$ elements $\sigma_{1}, \ldots, \sigma_{s-d}, \tau_{1}, \ldots, \tau_{d-1} \in y V$ such that $L$ is algebraic over $F:=k\left(y, \sigma_{1}, \ldots, \sigma_{s-d}, \tau_{1}, \ldots, \tau_{d-1}\right)$.

Set $K:=k\left(y, \sigma_{1}, \ldots, \sigma_{s-d}\right)$ and $R:=V \cap K$. Then $R$ is a DVR and the (y)-adic completion of $R$ is $R^{*}=k[[y]]$. In the notation of $1.5, B_{0}:=R\left[\tau_{1}, \ldots, \tau_{d-1}\right]_{\left(y, \tau_{1}, \ldots, \tau_{d-1}\right)}$ is a $d$-dimensional regular local ring and $V_{0}:=V \cap F$ is a DVR that birationally 
dominates $B_{0}$ and has $y$-adic completion $\widehat{V}_{0}=k[[y]]$. The following diagram displays these domains:

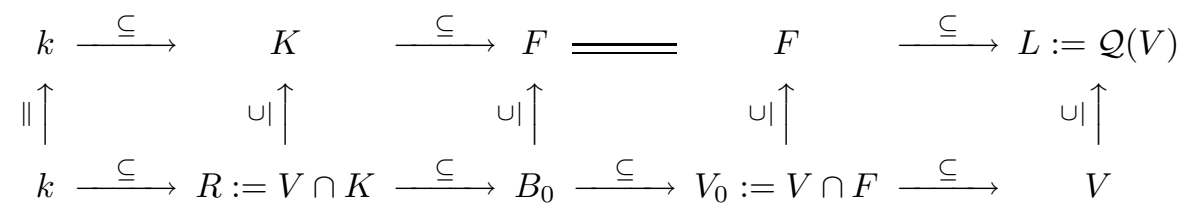

Let $\tau_{i n}$ be the $n$ th-endpiece of $\tau_{i}$ for each $i$; since $V_{0}=F \cap k[[y]]$, the $\tau_{\text {in }} \in V_{0}$ and $B_{n}=R\left[\tau_{1 n}, \ldots, \tau_{d-1, n}\right]_{\left(y, \tau_{1 n}, \ldots, \tau_{d-1, n}\right)}$ is, for each $n \in \mathbb{N}$, the first quadratic transform of $B_{n-1}$ along $V_{0}$.

Now $R_{y}^{*}$ is a field and thus is flat as an $R\left[\tau_{1}, \ldots, \tau_{d-1}\right]$-module. By Theorem 1.6, $V_{0}=\bigcup_{n=1}^{\infty} B_{n}$. An alternate way to justify this description of $V_{0}$ is to use the statement 1.1(4) where the $R$ of 1.1(4) is $B_{0}$, the $V$ of 1.1(4) is $V_{0}$, and each $R_{n}=B_{n}$. We have

$$
V_{0} \underset{\mathrm{alg}}{\longrightarrow} V \longrightarrow k[[y]]
$$

where $V_{0}$ and $V$ are DVRs of characteristic zero having completion $k[[y]]$. Since $V_{0}$ and $V$ are excellent, their Henselizations $V_{0}^{h}$ and $V^{h}$ are the set of elements of $k[[y]]$ algebraic over $V_{0}$ or $V$ [N2, (44.3)]. Thus $V_{0}^{h}=V^{h}$ and $V$ is a directed union of étale extensions of $V_{0}[\underline{\mathrm{R}},(1.3)]$.

2.1 Proposition. Let $B_{n}^{h}$ denote the Henselization of $B_{n}$. Then $\bigcup_{n=1}^{\infty} B_{n}^{h}=V_{0}^{h}=$ $V^{h}$.

Proof. The $\operatorname{ring} C:=\bigcup B_{n}^{h}$ is Henselian and contains $V_{0}$, so $V_{0}^{h}=V^{h} \subseteq C$. Moreover, the inclusion map $V \rightarrow C=\bigcup B_{n}^{h}$ extends to a map $V^{h} \stackrel{\sigma}{\rightarrow} C=\bigcup B_{n}^{h}$.

On the other hand, the maps $B_{n} \rightarrow V$ extend to maps $B_{n}^{h} \rightarrow V^{h}$ yielding a map $\rho: C \rightarrow V^{h}$ with $\sigma \rho=1_{C}$, and $\rho \sigma=1_{V^{h}}$. Thus $\bigcup_{n=1}^{\infty} B_{n}^{h}=V^{h}$.

Continuation of the proof of the main theorem. First assume $L$ is finitely generated over $k$. It follows that $L$ is finite algebraic over $F$. Since $\bigcup_{n=1}^{\infty} B_{n}^{h}=V^{h}$, we have $\bigcup_{n=1}^{\infty} \mathcal{Q}\left(B_{n}^{h}\right)=\mathcal{Q}\left(V^{h}\right)$ and $L \subseteq \mathcal{Q}\left(V^{h}\right)$. Since $L / F$ is finite algebraic, $L \subseteq \mathcal{Q}\left(B_{n}^{h}\right)$ for all sufficiently large $n$. By relabeling, we may assume $L \subseteq \mathcal{Q}\left(B_{n}^{h}\right)$ for all $n$. Let $C_{n}:=B_{n}^{h} \cap L$. Since $B_{n}$ is a regular local ring, $C_{n}$ is a regular local ring with $C_{n}^{h}=B_{n}^{h}[\mathrm{R},(1.3)]$.

Claim. For every $n, C_{n+1}$ dominates $C_{n}$ and $V$ dominates $C_{n}$. Also $\bigcup_{n=1}^{\infty} C_{n}=V$.

Proof of the claim. Since $B_{n+1}$ dominates $B_{n}$, we see that $B_{n+1}^{h}$ dominates $B_{n}^{h}$ and hence $C_{n+1}=B_{n+1}^{h} \cap L$ dominates $C_{n}=B_{n}^{h} \cap L$. Since $C_{n}=B_{n}^{h} \cap L \subseteq V^{h} \cap L=V$, it follows that $V$ dominates $C_{n}$ and $V_{0} \subseteq \bigcup_{n=1}^{\infty} C_{n} \subseteq V$. Since $V$ birationally dominates $\bigcup_{n=1}^{\infty} C_{n}$, it suffices to show that $\bigcup_{n=1}^{\infty} C_{n}$ is a DVR.

But by the same argument as before, $\bigcup_{n=1}^{\infty} C_{n}^{h}=\left(\bigcup_{n=1}^{\infty} C_{n}\right)^{h}=V^{h}$. This shows that $\bigcup_{n=1}^{\infty} C_{n}$ is a DVR, and therefore $\bigcup_{n=1}^{\infty} C_{n}=V$. Thus in the case where $L / k$ is finitely generated we have completed the proof of the main theorem including the "Moreover" statement.

2.2 Remark. An alternate approach to the definition of $C_{n}$ is as follows. Since $V$ is a directed union of étale extensions of $V_{0}$ and $\mathbb{Q}(V)=L$ is finite algebraic over $\mathbb{Q}\left(V_{0}\right)=F, V$ is étale over $V_{0}$ and therefore $V=V_{0}[\theta]=V_{0}[X] /(f(X))$, where $f(X)$ is a monic polynomial such that $f(\theta)=0$ and $f^{\prime}(\theta)$ is a unit of $V$. Let $B_{n}^{\prime}$ denote 
the integral closure of $B_{n}$ in $L$ and let $C_{n}=\left(B_{n}^{\prime}\right)_{\left(\mathbf{n} \cap B_{n}^{\prime}\right)}$. Since $\bigcup_{n=1}^{\infty} B_{n}=V_{0}$, it follows that $\bigcup_{n=1}^{\infty} C_{n}=V$. Moreover, for all sufficiently large $n, f(X) \in B_{n}[X]$ and $f^{\prime}(\theta)$ is a unit of $C_{n}$. Therefore $C_{n}$ is a regular local ring for all sufficiently large $n$ [N2, (38.6)]. As we note in Remark 2.3 below, this allows us to deduce a version of the main theorem also in the case where $k$ has characteristic $p>0$, provided the field $F$ can be chosen so that $L / F$ is separable.

Completion of the proof of the main theorem. If $L$ is not finitely generated over $k$, we choose a nested family of fields $L_{\alpha}$, with $\alpha \in \Gamma$, such that

(1) $F \subseteq L_{\alpha}$, for all $\alpha$.

(2) $L_{\alpha}$ is finite algebraic over $F$.

(3) $\bigcup_{\alpha \in \Gamma} L_{\alpha}=L$.

The rings $V_{\alpha}=L_{\alpha} \cap V$ are DVRs with $\bigcup_{\alpha \in \Gamma} V_{\alpha}=V$ and $V_{\alpha}^{h}=V^{h}$, since $V_{0} \subseteq V_{\alpha}$, for each $\alpha \in \Gamma$.

As above, $\bigcup_{n=1}^{\infty} B_{n}^{h}=V^{h}, \bigcup_{n=1}^{\infty} \mathcal{Q}\left(B_{n}^{h}\right)=\mathcal{Q}\left(V^{h}\right)$ and $L \subseteq \mathcal{Q}\left(V^{h}\right)$. Thus we see that for each $\alpha \in \Gamma$, there is an $n_{\alpha} \in \mathbb{N}$ such that $L_{\alpha} \subseteq \mathcal{Q}\left(B_{n}^{h}\right)$ for all $n \geq n_{\alpha}$.

Put $C_{n}^{(\alpha)}=L_{\alpha} \cap B_{n}^{h}$ for each $n \geq n_{\alpha}$. Then $V_{\alpha}=\bigcup_{n=n_{\alpha}}^{\infty} C_{n}^{(\alpha)}$ and $V_{\alpha}$ birationally dominates $C_{n}^{(\alpha)}$. Hence

$$
V=\bigcup_{\alpha \in \Gamma, n \geq n_{\alpha}} C_{n}^{(\alpha)}
$$

This completes the proof of the main theorem.

2.3 Remark. If the characteristic of $k$ is $p>0$, then the Henselization $V_{0}^{h}$ of $V_{0}=$ $F \cap k[[y]]$ may not equal the Henselization $V^{h}$ of $V=L \cap k[[y]]$, because the algebraic field extension $L / F$ may not be separable. But in the case where $L / F$ is separable algebraic, the fact that the DVRs $V$ and $V_{0}$ have the same completion implies that $V$ is a directed union of étale extensions of $V_{0}$ (see, for example, [AH, Theorem 2.7]). Therefore in the case where $L / F$ is separable algebraic, $V$ is a directed union of regular local rings of dimension $d$.

Thus for a local domain $(R, \mathbf{m})$ essentially of finite type over a field $k$ of characteristic $p>0$, a result analogous to Corollary 1.3 is true provided there exists a subfield $F$ of $L$ such that $F$ is purely transcendental over $k, L / F$ is separable algebraic, and $F$ contains a generator for the maximal ideal of $V$.

In characteristic $p>0$, with $V$ excellent and the extension separable, the ring $V_{0}$ need not be excellent (see for example [HRS, (3.3) and (3.4)]).

\section{REFERENCES}

[A] S. Abhyankar, On the valuations centered in a local domain, Amer. J. Math. 78 (1956), 321-348. MR 18:556b

[AH] S. Abhyankar and W. Heinzer, Ramification in infinite integral extensions, J. Algebra 170 (1994), 861-879. MR 95k:13006

[C1] S. Cutkosky, Local factorization of birational maps, Adv. Math. 132 (1997), 167-315. MR 99c:14018

[C2] Local factorization and monomialization of morphisms, to appear.

[HRS] W. Heinzer, C. Rotthaus and J. Sally, Formal fibers and birational extensions, Nagoya Math J. 131 (1993), 1-38. MR 95a:13008

[HRW1] W. Heinzer, C. Rotthaus and S. Wiegand, Noetherian rings between a semilocal domain and its completion, J. Algebra 198 (1997), 627-655. MR 99a:13008 
[HRW2] — Building Noetherian domains inside an ideal-adic completion, Abelian Groups, Module Theory and Topology Proceedings in Honor of Adalberto Orsatti's 60th Birthday (D. Dikranjan and L. Salce, ed.), Dekker Inc, 1998, pp. 279-287. MR 99m:13035

[HRW3] , Noetherian domains inside a homomorphic image of a completion, J. Algebra 215 (1999), 666-681. CMP 99:12

[M1] H. Matsumura, Commutative Ring Theory, Cambridge University Press, Cambridge, 1986. MR 88h:13001

[M2] - Commutative Algebra, second edition, Benjamin/Cummings, Reading, MA, 1980. MR 82i:13003

[N1] M. Nagata, An example of a normal local ring which is analytically reducible, Mem. Coll. Sci., Univ. Kyoto 31 (1958), 83-85. MR 20:3864

[N2] L Local Rings, John Wiley, 1962. MR 27:5790

[R] C. Rotthaus, Homomorphic images of regular local rings, Comm. in Alg. 24 (1996), 445-476. MR 97b:13029

[S] D. Shannon, Monoidal transforms of regular local rings, Amer. J. Math. 95 (1973), 284-320. MR 48:8492

[Z1] O. Zariski, Local uniformization on algebraic varieties, Ann. Math. 41 (1940), 852-896. MR 2:124a

[Z2] - Applicazioni geometriche della teoria delle valutazioni, Rend. Circolo Mat. Palermo 13(5) (1954), 1-38. MR 16:165f

[ZS] O. Zariski and P. Samuel, Commutative Algebra vol II, Van Nostrand, Princeton, NJ, 1960. MR 22:11006

Department of Mathematics, Purdue University, West Lafayette, Indiana 47907-1395

E-mail address: heinzer@math.purdue.edu

Department of Mathematics, Michigan State University, East Lansing, Michigan 48824-1027

E-mail address: rotthaus@math.msu.edu

Department of Mathematics and Statistics, University of Nebraska, Lincoln, NeBRASKA 68588-0323

E-mail address: swiegand@math.unl.edu 